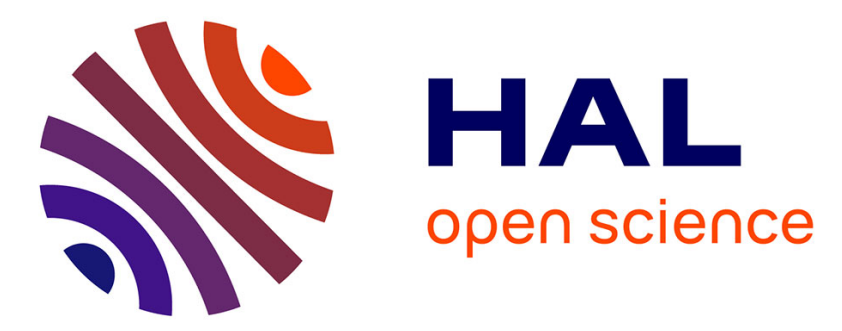

\title{
Dialogue essais-modèle pour l'interprétation des liens entre mesure d'auto-échauffement sous chargement cyclique et fatigue polycyclique
}

Sylvain Calloch, Cédric Doudard, François Hild, Martin Poncelet, Bastien Weber, André Galtier

\section{To cite this version:}

Sylvain Calloch, Cédric Doudard, François Hild, Martin Poncelet, Bastien Weber, et al.. Dialogue essais-modèle pour l'interprétation des liens entre mesure d'auto-échauffement sous chargement cyclique et fatigue polycyclique. Revue de Métallurgie, 2010, 107 (2-3), pp.75-82. 10.1051/metal/2010010. hal-00521207

\section{HAL Id: hal-00521207 \\ https://hal.science/hal-00521207}

Submitted on 26 Sep 2010

HAL is a multi-disciplinary open access archive for the deposit and dissemination of scientific research documents, whether they are published or not. The documents may come from teaching and research institutions in France or abroad, or from public or private research centers.
L'archive ouverte pluridisciplinaire HAL, est destinée au dépôt et à la diffusion de documents scientifiques de niveau recherche, publiés ou non, émanant des établissements d'enseignement et de recherche français ou étrangers, des laboratoires publics ou privés. 
Dialogue essais-modèle pour l'interprétation des liens entre mesure d'autoéchauffement sous chargement cyclique et fatigue polycyclique

Model-experiment dialogue to link self-heating under cyclic loadings to high cycle fatigue properties

SYLVAIN CALLOCH ${ }^{1 *}$, CEDRIC DOUDARD ${ }^{1}$, FRANÇOIS HILD ${ }^{2}$, MARTIN PONCELET ${ }^{2}$, BASTIEN WEBER ${ }^{3}$ et ANDRÉ GALTIER ${ }^{4}$

${ }^{1}$ LBMS, ENSIETA/UBO/ENIB, 2 rue François Verny, 29806 Brest, France

${ }^{2}$ LMT-Cachan, 61 avenue du Président Wilson, 94235 Cachan, France

${ }^{3}$ ArcelorMittal, BP 3032, Maizières-lès-Metz, France

${ }^{4}$ CETIM, 52 avenue Félix Louat, BP 8067, 60304 Senlis, France

${ }^{*}$ Corresponding author. Tel.: +33 2983487 23; Fax: +33 298348730 
Résumé : Une démarche de développement d'un modèle probabiliste à deux échelles pour la fatigue HCF des aciers est proposée. Elle est basée sur l'utilisation de mesure d'auto-échauffement sous chargements cycliques et est validée sur la base de la prévision des courbes de Wöhler d'un acier dual-phase pour différents rapport de charge.

\begin{abstract}
It is proposed to develop a probabilistic two-scale model for high cycle fatigue. It is based on the using of self-heating measurements under cyclic loadings. The validation of this approach is obtained by predicting $S / N$ curves for different mean stress of a dual-phase steel.
\end{abstract}




\section{INTRODUCTION}

La fatigue polycyclique (i.e., fatigue à grand nombre de cycles ou fatigue $H C F$ ) est un aspect du comportement mécanique des matériaux métalliques particulièrement insidieux du fait de son caractère progressif et masqué. De plus, c'est un phénomène complexe dans la mesure où il dépend d'un grand nombre de paramètres : composition chimique et microstructure du matériau, taille de la pièce, amplitude et multiaxialité du chargement mécanique, environnement, température,... Au cours des dernières années, des avancées significatives ont pu être faites, en ce qui concerne aussi bien le calcul des pièces à la fatigue que la compréhension des mécanismes microstructuraux qui contrôlent la dégradation des matériaux sous sollicitations répétées. Cependant, des verrous scientifiques subsistent. L'une des raisons majeures est le coût (en temps et en argent) des essais traditionnels de fatigue (i.e., il faut plus d'une semaine d'essais ininterrompus et plusieurs dizaines d'éprouvettes pour obtenir une courbe de Wöhler). On comprend alors que cela représente un frein à l'étude et à la modélisation de l'influence de tel ou tel paramètre sur les propriétés à la fatigue des matériaux et des structures.

Depuis quelques années, un certain nombre d'équipes de recherche travaille sur la détermination des propriétés à la fatigue à grand nombre de cycles des matériaux métalliques à partir d'essais d'auto-échauffement [1-6]. Cette méthode est basée sur l'observation de l'évolution de la température moyenne stabilisée d'une éprouvette soumise à une séquence de chargements cycliques par blocs. Lors de ce type d'essais, on observe, une fois passé un certain niveau de chargement, que la température moyenne stabilisée de l'éprouvette augmente de façon significative. Cette augmentation de température est reliée au fait que la limite de fatigue du matériau a été dépassée donnant naissance à des mécanismes dissipatifs de micro-plasticité. Une exploitation empirique de ces essais montre qu'ils permettent d'estimer de façon rapide (i.e., à partir d'un seul essai 
d'une durée d'une heure et ne nécessitant qu'une seule éprouvette) la limite d'endurance du matériau [2-3]. Plus récemment, le développement d'une méthode d'analyse à partir d'un modèle probabiliste à deux échelles, a démontré que les essais d'auto-échauffement permettent, avec un nombre d'éprouvettes réduit, de déterminer non seulement la limite d'endurance moyenne d'un acier, mais également de prévoir la dispersion des résultats des campagnes d'essais classiques de fatigue [6].

Nous proposons, dans ce papier, de montrer la méthode de développement d'un modèle probabiliste à deux échelles pour la fatigue $H C F$ en nous appuyant essentiellement sur des observations expérimentales issues d'essais d'auto-échauffement. Ce papier est donc organisé en trois parties. Dans la première, nous présentons un certain nombre de résultats d'essais d'auto-échauffement sous chargement cyclique. Chaque résultat nous donnera des pistes concernant les ingrédients à introduire dans la modélisation. Dans la deuxième partie, une version du modèle probabiliste à deux échelles pour les aciers est présentée. Enfin, dans la troisième partie, en nous appuyant sur la prévision de propriétés à la fatigue $H C F$, le modèle est validé.

\section{AUTO-ECHAUFFEMENT SOUS CHARGEMENTS CYCLIQUES}

Les ingrédients majeurs introduits dans le modèle que nous proposons sont, d'une part, l'échelle de description de l'activité micro-plastique responsable de l'amorçage des mécanismes d'endommagement en fatigue, d'autre part, le scénario d'activation des sites de micro-plasticité et enfin la description du comportement mécanique de ces inclusions élasto-plastiques. Nous allons voir dans cette partie de quelle manière l'observation de l'auto-échauffement sous chargement cyclique peut nous donner des renseignements précieux sur les trois ingrédients majeurs de notre modélisation. 


\section{Évolution de la température sous amplitude constante}

La séquence de base d'un essai d'auto-échauffement consiste à appliquer un chargement de traction-compression cyclique caractérisé par son amplitude, $\Sigma_{0}$, son rapport de charge, $R$, et sa fréquence, $f_{r}$ et d'enregistrer l'évolution de sa température au cours des cycles. Les figures $1 \mathrm{a}$ et $1 \mathrm{~b}$ montrent, pour deux matériaux différents, l'évolution de la variation de température moyenne, $\theta=\left(T-T_{r e f}\right)$, en fonction du temps. On peut voir que, suivant le matériau, l'état d'équilibre thermique peut varier ou non avec le nombre de cycles (i.e., la dissipation intrinsèque du matériau évolue ou non au cours des cycles). Ce type d'observation expérimentale nous renseignera sur la nature de l'écrouissage des sites élasto-plastiques ainsi que sur leur évolution [7].
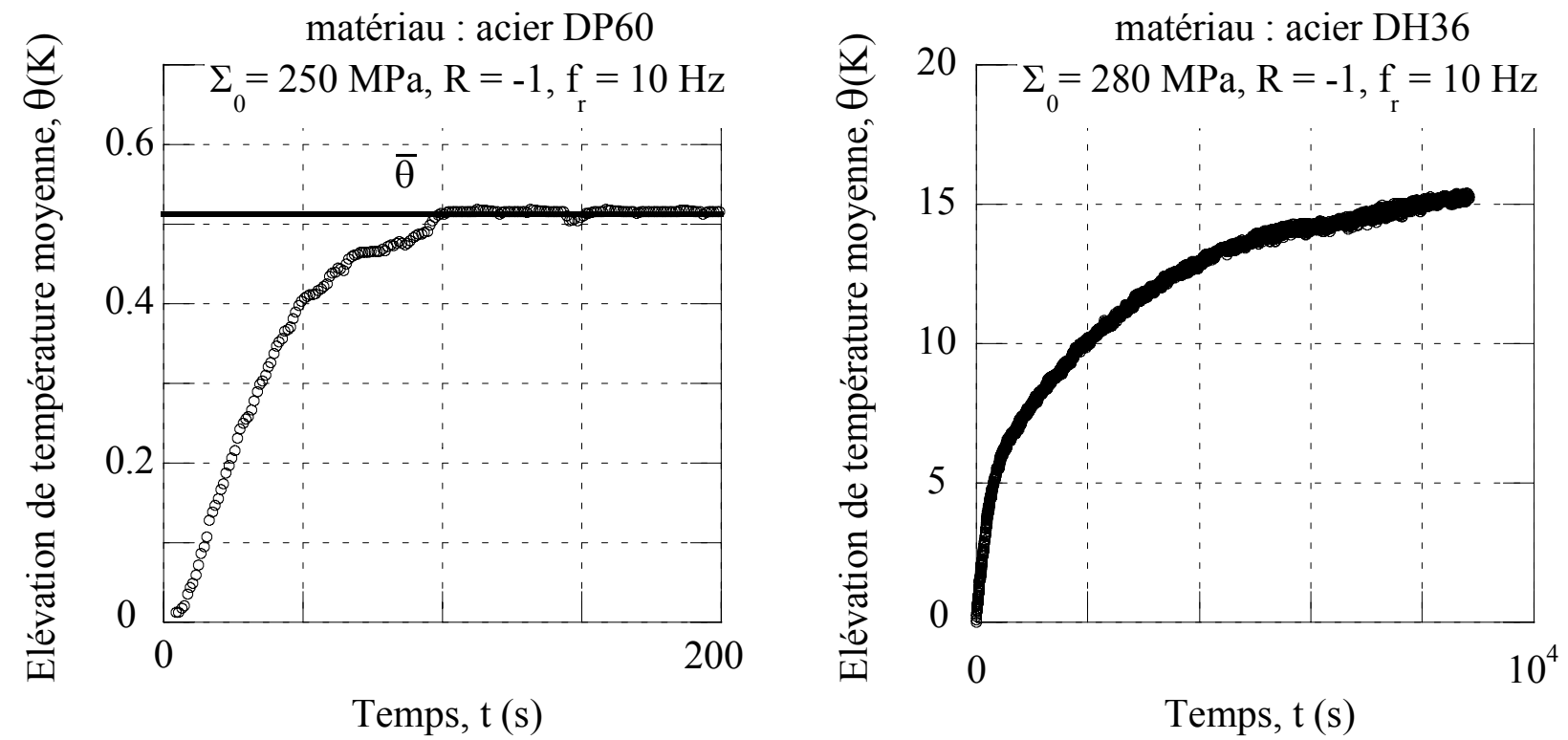

Fig. 1. Évolution de la température de l'éprouvette sous chargement cyclique à amplitude constante. -a- cas d'un acier dual-phase $\left(\Sigma_{0}=250 \mathrm{MPa}\right)$, -b- cas de l'acier DH36

$$
\left(\Sigma_{0}=280 \mathrm{MPa}\right) \text {. }
$$

Fig. 1. Change of the temperature variation under cyclic loadings: -a-dual-phase steel

$$
\left(\Sigma_{0}=250 \mathrm{MPa}\right),-b-\mathrm{DH} 36 \text { steel }\left(\Sigma_{0}=280 \mathrm{MPa}\right) \text {. }
$$




\section{Influence de l’amplitude du chargement}

La figure 2 montre l'évolution de la température stabilisée, $\bar{\theta}$, en fonction de l'amplitude du chargement dans le cas de l'acier dual-phase. On peut voir que cette température augmente de façon significative à partir d'un certain niveau d'amplitude. Ce type d'observation nous renseignera sur les dispersions auxquelles on peut s'attendre lors d'essais classiques de fatigue $[6,8]$.

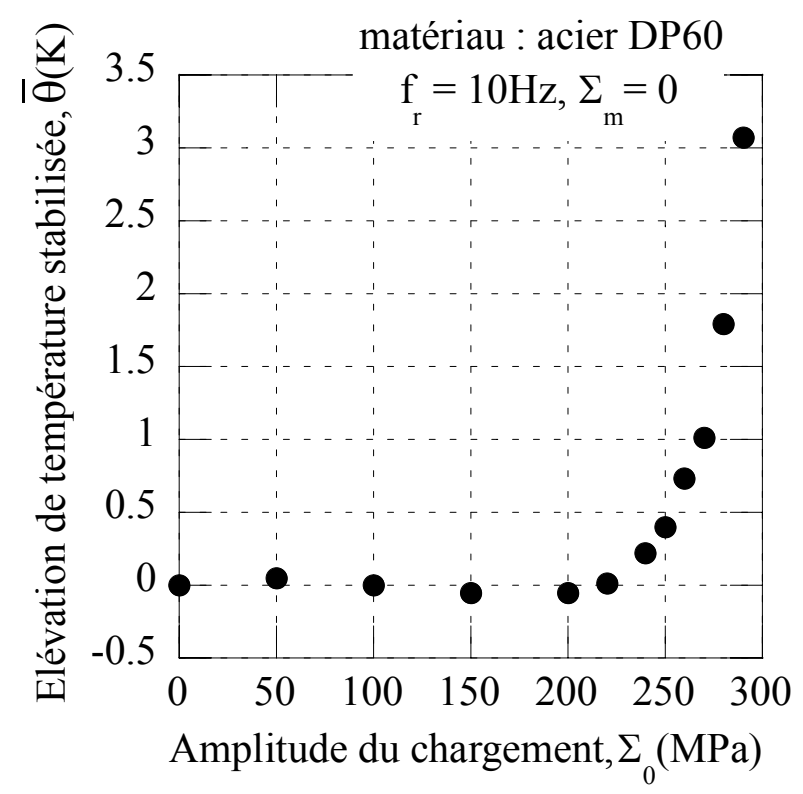

Fig. 2. Courbes d'auto-échauffement sous chargement cyclique d'un acier dual-phase.

Fig. 2. Self-heating curve under cyclic loadings for a dual-phase steel.

\section{Influence de la fréquence du chargement}

La figure 3 montre l'influence de la fréquence du chargement, $f_{r}$, sur la courbe d'autoéchauffement. On peut voir que, dans le cas de l'acier dual-phase, la température moyenne stabilisée dépend linéairement de la fréquence du chargement. Ce type d'observation nous donne des renseignements sur la nature du comportement mécanique des sites dissipatifs (e.g., élasto-plastique, élasto-viscoplastique...). 


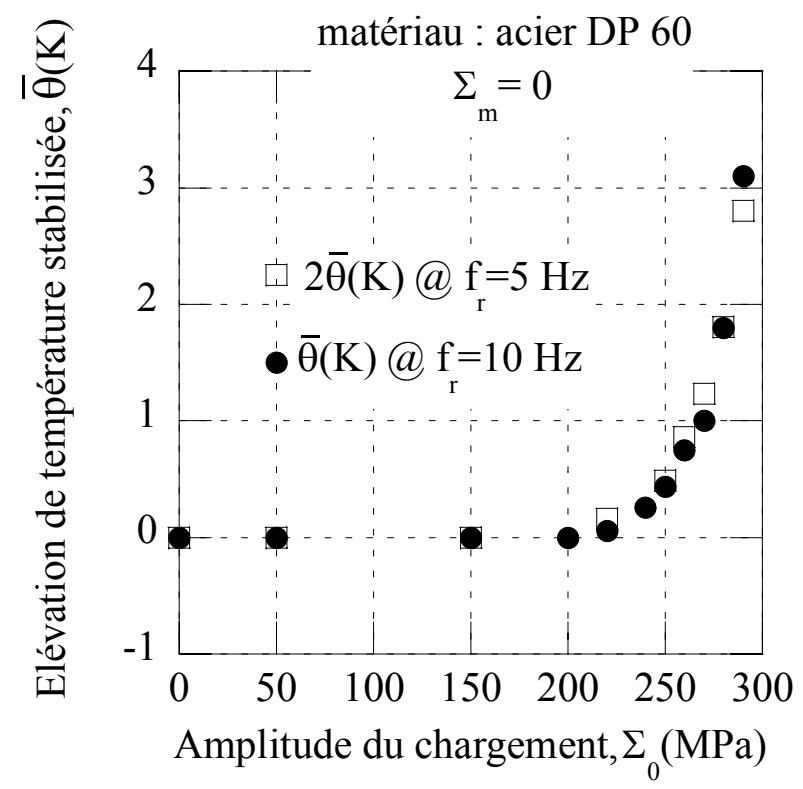

Fig. 3. Influence de la fréquence du chargement sur la courbe d'auto-échauffement d'un acier dual-phase.

Fig. 3. Influence of the loading frequency on the self-heating curve for a dual-phase steel.

\section{Influence du rapport de charge}

La figure 4 montre l'influence du rapport de charge, $R=\Sigma_{\min } / \Sigma_{\max }$, sur la courbe d'autoéchauffement. Ce type d'observation nous donne des renseignements sur le scénario d'activation des sources de dissipation [9].

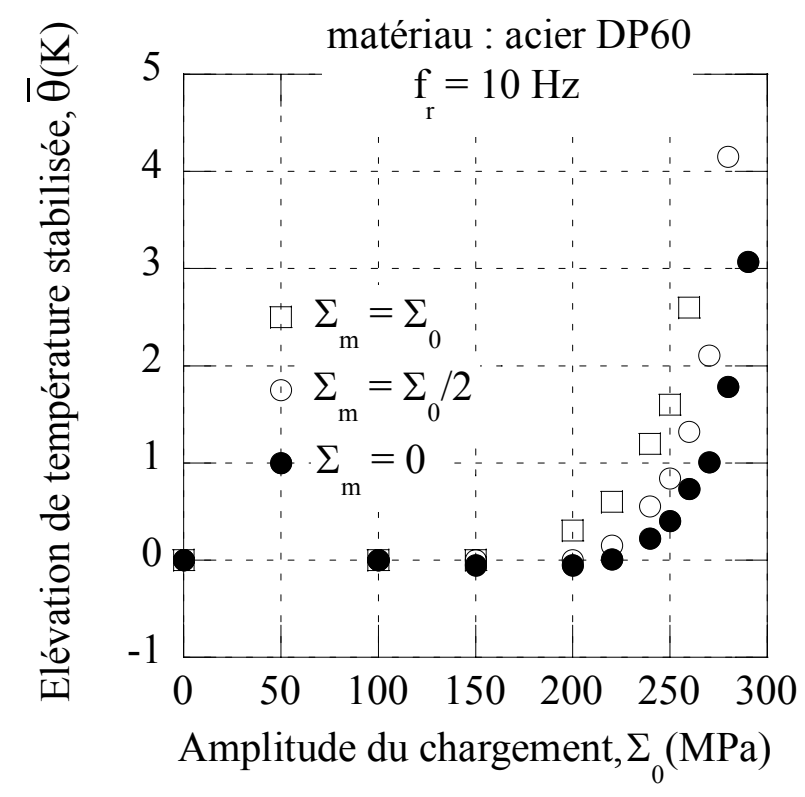


Fig. 4. Influence du rapport de charge sur la courbe d'auto-échauffement d'un acier dualphase.

Fig. 4. Influence of the mean stress on the self-heating curve for a dual-phase steel.

\section{Auto-échauffement sous chargement multiaxial cyclique}

Les figures $5 \mathrm{a}$ et $5 \mathrm{~b}$ montrent deux surfaces d'iso-auto-échauffement obtenues respectivement sous sollicitations cycliques de bi-traction-compression $[9,10,11]$ et de traction-torsion $[10,12]$. Il s'agit de représenter pour chaque direction dans le plan des contraintes lié à chaque essai l'amplitude de chargement conduisant à un autoéchauffement donné $\left(\bar{\theta}_{\text {off }}\right)$. Ces essais nous renseignent sur le comportement mécanique des sites dissipatifs sous chargement multiaxial.

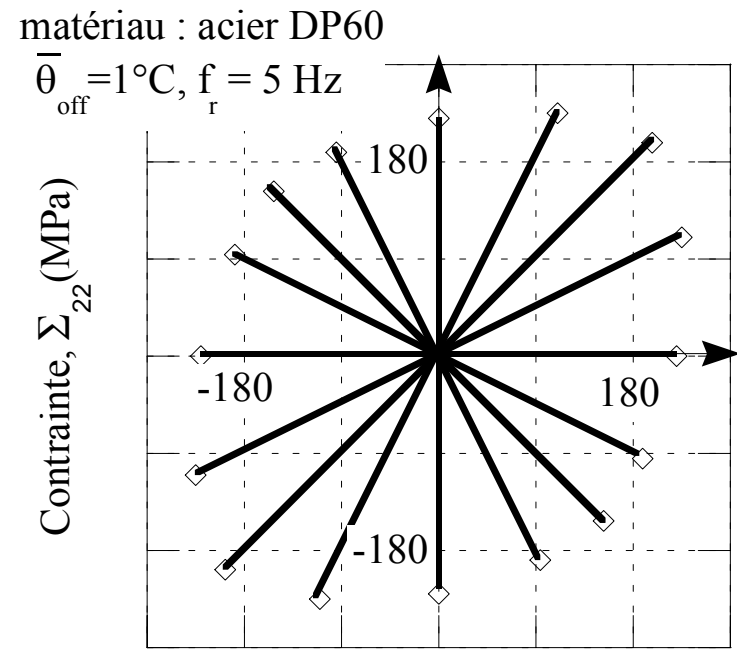

Contrainte, $\Sigma_{11}(\mathrm{MPa})$ matériau : acier $\mathrm{C} 45$

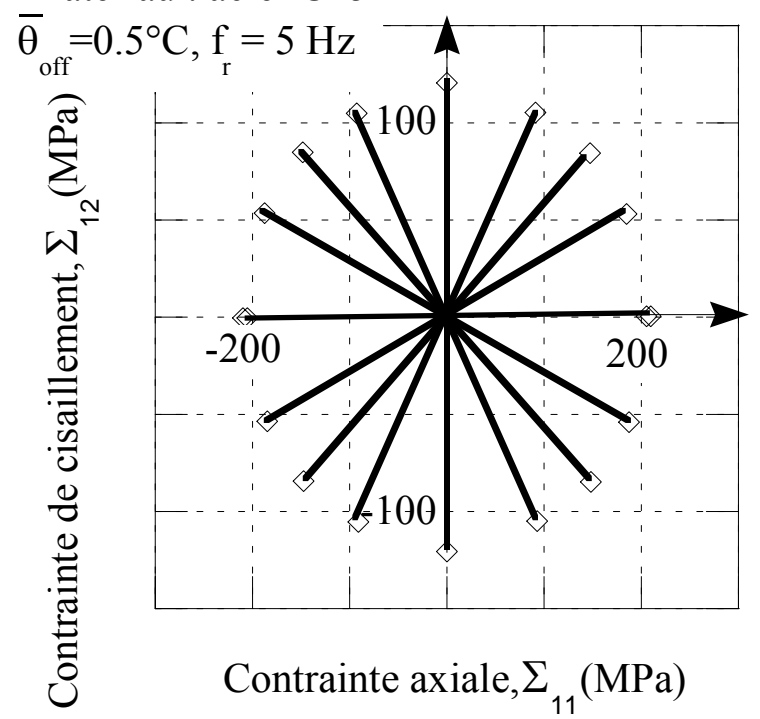

Fig. 5. Surfaces d'iso-auto-échauffement sous chargement cyclique multiaxial. -a- cas de la bitraction-compression, -b- cas de la traction-torsion.

Fig. 5. Iso-temperature surface under multiaxial cyclic loadings: - a-bi-tension-compression loadings, - $b$ - torsion-tension loadings.

\section{UN MODELE A DEUX ÉCHELLES POUR LA FATIGUE HCF}

L'ensemble des résultats d'essai présenté dans la partie précédente va nous permettre de proposer un modèle probabiliste à deux échelles pour la fatigue à grand nombre de cycles des matériaux métalliques. Il fait l'objet de cette troisième partie. 


\section{Scénario probabiliste de l’apparition de la micro-plasticité [6-9]}

Le comportement à la fatigue $H C F$ des matériaux métalliques est décrit en considérant un ensemble d'inclusions élasto-plastiques (i.e., sites), de volume $V_{s}$, répartis de manière aléatoire dans une matrice à comportement élastique. Le scénario d'apparition des sites actifs (i.e., sites dans lesquels la micro-plasticité est active) est supposé être piloté par un processus ponctuel de Poisson. Ainsi, la probabilité de trouver $k$ sites actifs dans un domaine $\Omega$ de volume $V_{\Omega}$ est donnée par [13]

$$
\mathrm{P}_{\mathrm{k}}(\Omega)=\frac{[-\mathrm{N}(\Omega)]^{\mathrm{k}}}{\mathrm{k} !} \exp [-\mathrm{N}(\Omega)]
$$

où $N(\Omega)$ est le nombre moyen de sites actifs dans le domaine $\Omega$ donné par

$$
\mathrm{N}(\Omega)=\lambda \mathrm{V}_{\Omega}
$$

avec $\lambda$, la densité de sites actifs, donnée par

$$
\lambda(\Omega)=\frac{1}{\mathrm{~V}_{0}}\left(\frac{\Sigma_{0}^{\mathrm{eq}}\left(1+\alpha_{\mathrm{H}}\left(\mathrm{P}_{0}-1 / 3\right)\right)}{\mathrm{S}_{0}}\right)^{m},
$$

où $V_{0} S_{0}^{m}, m$ et $\alpha_{H}$ sont trois paramètres qui dépendent du matériau. On considère donc que la densité de sites actifs suit une loi puissance de l'amplitude des contraintes équivalente de von Mises $\Sigma_{0}^{\mathrm{eq}}=\operatorname{Max}_{t} J_{2}\left(\underline{\underline{\Sigma}}(t)-\underline{\underline{\Sigma_{m}}}\right)$ avec la contrainte moyenne, $\underline{\underline{\Sigma}}_{m}$, donneé par

$$
\underline{\underline{\Sigma}}_{m}=\underline{\underline{S}}_{m}+I_{1, \text { moy }} \underline{\underline{I}}=\operatorname{Min}_{Y}\left[\operatorname{Max}_{t} J_{2}(\underline{\underline{\Sigma}}(t)-\underline{\underline{Y}})\right]+\frac{1}{3} \operatorname{Min}_{z}[\operatorname{Max}(\operatorname{trace}(\underline{\underline{\Sigma}}(t))-z)] \underline{\underline{\underline{I}}},
$$

et le second invariant, $J_{2}$, définie par

$$
J_{2}\left(\underline{\underline{\Sigma}}(t)-\underline{\underline{\Sigma}}_{m}\right)=\sqrt{\frac{3}{2}\left(\underline{\underline{S}}-\underline{\underline{S}}_{m}\right):\left(\underline{\underline{S}}-\underline{\underline{S}}_{m}\right)},
$$

où $\underset{\underline{s}}{\underline{\sigma}} \underline{\underline{\sigma}}-\frac{1}{3} \operatorname{trace}(\underline{\underline{\sigma}}) \underline{\underline{I}}$ et $\underset{\underline{I}}{\underline{1}}$ le tenseur unité d'ordre 2 . 
La dépendance à la pression hydrostatique est introduite avec le facteur $\mathrm{P}_{0}=I_{1, \max } / \Sigma_{0}^{\mathrm{eq}}$ pour rendre compte de l'effet de la contrainte moyenne et de la multiaxialité des contraintes sur les propriétés à la fatigue et sur les mesures de l'auto-échauffement [10]. La figure 6 schématise le scénario d'apparition des sites de micro-plasticité dans un domaine de volume $V_{\Omega}$ pour un chargement de traction alternée (i.e., $\mathrm{R}=-1$ ).

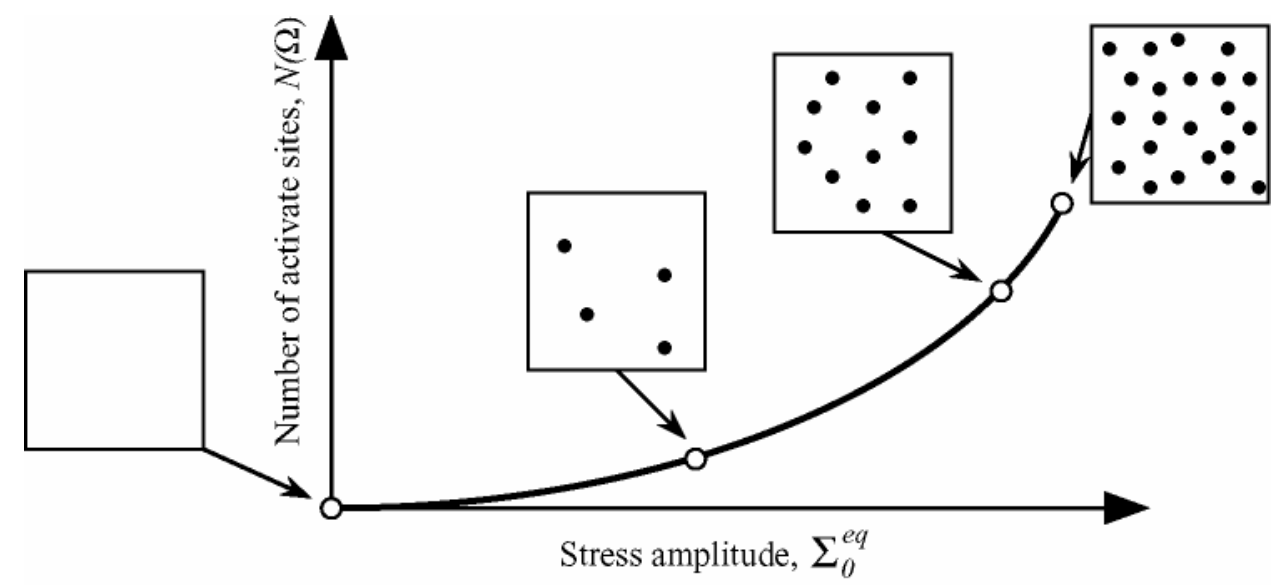

Fig. 6. Représentation schématique du scénario d'apparition de la micro-plasticité dans un domaine $\Omega$ en fonction de l'amplitude du chargement pour un chargement de tractioncompression alternée.

Fig. 6. Schematic view of the activation scenario of elastoplastic sites in a domain $\Omega$ with the stress amplitude for a alternate tension-compression test.

\section{Comportement élasto-plastique des sites actifs [6-9]}

Pour décrire le caractère dissipatif des sollicitations cycliques révélé par les essais d'autoéchauffement, l'état de contrainte dans les sites et leur comportement élasto-plastique doivent être décrits. Pour cela on utilise la loi de localisation suivante

$$
\underline{\underline{\sigma}}=\underline{\underline{\Sigma}}-2 \mu(1-\beta) \underline{\underline{\varepsilon}}^{\mathrm{p}}
$$

où $\beta=\frac{2(4-5 v)}{15(1-v)}$ et $\mu, v$ sont respectivement le module de cisaillement et le coefficient de Poisson [14]. Enfin le concept de la thermodynamique des milieux continus est utilisé pour modéliser le comportement des sites [15]. A chaque instant, t, l'état thermodynamique de chaque site est caractérisé par 4 variables d'état, à savoir, $\theta$, la variation de température, 
$\underline{\varepsilon}^{\mathrm{e}}$, la déformation élastique, $\underline{\varepsilon}^{\mathrm{p}}$, la déformation plastique et $\mathrm{p}$, la déformation plastique cumulée définie par

$$
\dot{\mathrm{p}}=\sqrt{\frac{2}{3} \underline{\dot{\varepsilon}}^{\mathrm{p}}: \dot{\underline{\varepsilon}}^{\mathrm{p}}}
$$

L'énergie libre spécifique d'Helmholtz de chaque site est donnée par

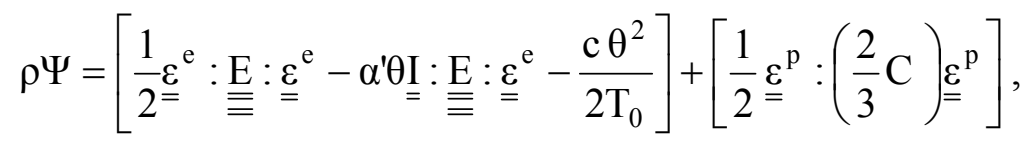

avec $\rho$ la masse volumique, $\alpha$ ' le coefficient de dilatation, c la chaleur spécifique, $\underline{\underline{\equiv}}$ le tenseur d'élasticité, C le paramètre de proportionnalité de l'écrouissage cinématique linéaire. Un écrouissage mixte (cinématique et isotrope) doit être considéré dans le cas où l'équilibre thermique varie au cours des cycles [7]. Par souci de simplicité, on se limite ici au cas où cet équilibre n'évolue pas (cas de l'acier dual-phase). Par conséquent l'écrouissage cinématique est relié à la déformation plastique par

$$
\underline{\underline{X}}=\frac{2}{3} \mathrm{C} \underset{\underline{\varepsilon}}{\underline{p}}
$$

La loi de normalité est considérée pour décrire l'évolution de la déformation plastique

$$
\underline{\dot{\varepsilon}}^{\mathrm{p}}=\dot{\lambda} \frac{\partial \mathrm{f}}{\partial \underline{\underline{\sigma}}}
$$

où $\dot{\lambda}$ est le multiplicateur plastique et $\mathrm{f}$ la surface seuil définie par

$$
\mathrm{f}=\sqrt{\frac{3}{2}(\underline{\underline{s}}-\underline{\underline{X}}):(\underline{\underline{s}}-\underline{\underline{X}})}-\sigma_{\mathrm{y}}-\mathrm{R} \leq 0
$$

où $\sigma_{\mathrm{y}}$ est la imite d'élasticité du site considéré. On en déduit alors que la dissipation intrinsèque cyclique pour un site de limite, $\sigma_{\mathrm{y}}$, et un chargement d'amplitude équivalente, $\Sigma_{0}^{\mathrm{eq}}$, est donnée par 


$$
\mathrm{D}_{\text {site }}\left(\Sigma_{0}^{\mathrm{eq}}, \sigma_{\mathrm{y}}\right)=\int_{\text {cycle }}\left((\underline{\underline{\sigma}}-\underline{\underline{X}}): \underline{\underline{\dot{\varepsilon}}}^{\mathrm{p}}\right) \mathrm{V}_{\mathrm{S}} \mathrm{dt}=\frac{4 \mathrm{~V}_{\mathrm{S}} \sigma_{\mathrm{y}}}{\mathrm{h}}\left\langle\Sigma_{0}^{\mathrm{eq}}-\sigma_{\mathrm{y}}\right\rangle,
$$

où $\mathrm{h}=C+3 \mu(1-\beta),\langle$.$\rangle sont les crochets de Macauley (i.e. la part positive de '.'). Pour$ un domaine $\Omega$ de volume $V_{\Omega}$ la dissipation cyclique volumique, $\Delta$, est obtenue par calcul de la moyenne de la dissipation des différents sites actifs dans ce domaine

$$
\Delta=\int_{0}^{\Sigma_{0}^{\mathrm{eq}}} \mathrm{D}_{\text {site }}\left(\Sigma_{0}^{\mathrm{eq}}, \Sigma\right) \frac{\mathrm{d} \lambda(\Sigma)}{\mathrm{d} \Sigma} \mathrm{d} \Sigma=\frac{4 \mathrm{mV}_{0}}{\mathrm{~h}(\mathrm{~m}+1)(\mathrm{m}+2)} \frac{\left(\Sigma_{0}^{\mathrm{eq}}\right)^{\mathrm{m}+2}\left(1+\alpha_{\mathrm{H}}\left(\mathrm{P}_{0}-1 / 3\right)\right)^{\mathrm{m}}}{\mathrm{V}_{0}\left(\mathrm{~S}_{0}\right)^{\mathrm{m}}},
$$

\section{Description des courbes d'auto-échauffement [6-9]}

Toujours dans le cadre proposé, les courbes d'auto-échauffement sont décrites à partir de la résolution de l'équation de la chaleur [9]

$$
\dot{\theta}+\frac{\theta}{\tau_{e q}}=\frac{f_{r} \quad 4 m V_{0}}{\rho c h(m+1)(m+2)} \frac{\left(\Sigma_{0}^{\mathrm{eq}}\right)^{\mathrm{m}+2}\left(1+\alpha_{\mathrm{H}}\left(\mathrm{P}_{0}-1 / 3\right)\right)^{\mathrm{m}}}{\mathrm{V}_{0}\left(\mathrm{~S}_{0}\right)^{\mathrm{m}}},
$$

où $\tau_{e q}$ est un temps caractéristique qui dépend des conditions aux limites thermiques. La solution stabilisée, $\bar{\theta}$, en fonction de $\Sigma_{0}^{e q}$ est

$$
\bar{\theta}=\frac{4 f_{r} \tau_{e q} m V_{0}}{\rho \operatorname{ch}(m+1)(m+2)} \frac{\left(\Sigma_{0}^{\mathrm{eq}}\right)^{\mathrm{m}+2}\left(1+\alpha_{\mathrm{H}}\left(\mathrm{P}_{0}-1 / 3\right)\right)^{\mathrm{m}}}{\mathrm{V}_{0}\left(\mathrm{~S}_{0}\right)^{\mathrm{m}}}
$$

L'équation (15) traduit bien la dépendance linéaire à la fréquence du chargement observée sur Fig. 3.

\section{Description des courbes de Wöhler [6-9]}

Nous allons voir dans cette partie comment, tout en conservant le cadre proposé jusque là, la fatigue à grand nombre de cycles peut être abordée. Nous distinguons les cas de la fatigue à endurance illimitée de celle à endurance limitée. Dans le premier cas, la théorie du maillon le plus faible est considérée [16]. Alors, on peut montrer que la limité 
d'endurance moyenne, $\bar{\Sigma}_{\infty}$, et son écart type, $\overline{\bar{\Sigma}}_{\infty}$, sont donnés, dans le cas où les contraintes sont homogènes, par [9]

$$
\left\{\begin{array}{l}
{\overline{\left(\Sigma_{0}^{\mathrm{eq}}\right)_{\infty}}}_{\infty} \bar{\Sigma}_{\infty}=S_{0}\left(1+\alpha_{\mathrm{H}}\left(\mathrm{P}_{0}-\frac{1}{3}\right)\right)^{-2}\left(\frac{V_{0}}{V_{\Omega}}\right)^{\frac{1}{m}} \Gamma\left(1+\frac{1}{m}\right) \\
\overline{\bar{\Sigma}}_{\infty}^{2}=S_{0}^{2}\left(1+\alpha_{\mathrm{H}}\left(\mathrm{P}_{0}-\frac{1}{3}\right)\right)^{-2}\left(\frac{V_{0}}{V_{\Omega}}\right)^{\frac{2}{m}}\left[\Gamma\left(1+\frac{2}{m}\right)-\Gamma^{2}\left(1+\frac{1}{m}\right)\right]
\end{array}\right.
$$

où la fonction $\Gamma(p)$ définie par

$$
\Gamma(p)=\int_{0}^{\infty} t^{p-1} \exp (-t) d t
$$

Les équations (14) correspondent au modèle de Weibull [17]. Dans le cas de l'endurance limitée, un critère de fatigue basé sur le concept d'énergie dissipée critique est retenu. Le nombre de cycles à rupture est alors relié à un paramètre dépendant du matériau, $A$, et à la limite d'endurance, $\Sigma_{\infty}\left(P_{F}\right)$, par la relation [9]

$$
N=\frac{A}{\Sigma_{\infty}\left(P_{F}\right)\left\langle\Sigma_{0}^{e q}-\Sigma_{\infty}\left(P_{F}\right)\right\rangle},
$$

où $\Sigma_{\infty}\left(P_{F}\right)$ est défini par

$$
\frac{\ln \left(1-P_{F}\right)}{\ln (1-0.5)}=\left[\frac{\Sigma_{\infty}\left(P_{F}\right)}{\Sigma_{\infty}(0.5)}\right]^{m},
$$

$\operatorname{avec} \Sigma_{\infty}(0.5)=\bar{\Sigma}_{\infty}\left[\ln (2)^{1 / m} / \Gamma\left(1+\frac{1}{m}\right)\right]$.

\section{PREVISION DES COURBES DE WÖHLER}

L'identification des 4 paramètres du modèle présenté précédemment, à savoir $V_{0} S_{0}^{m}, m$, $\alpha_{m}$ et $\mathrm{A}$, n'est basé que sur l'utilisation de 2 essais d'auto-échauffement, à rapport de charge différent, mené jusqu'à rupture lors du dernier palier [9]. Les figures $7 \mathrm{a}$ et $7 \mathrm{~b}$ 
illustrent l'étape d'identification des paramètres dans le cas d'un acier dual phase. Les valeurs des paramètres identifiés sont données dans le tableau 1.
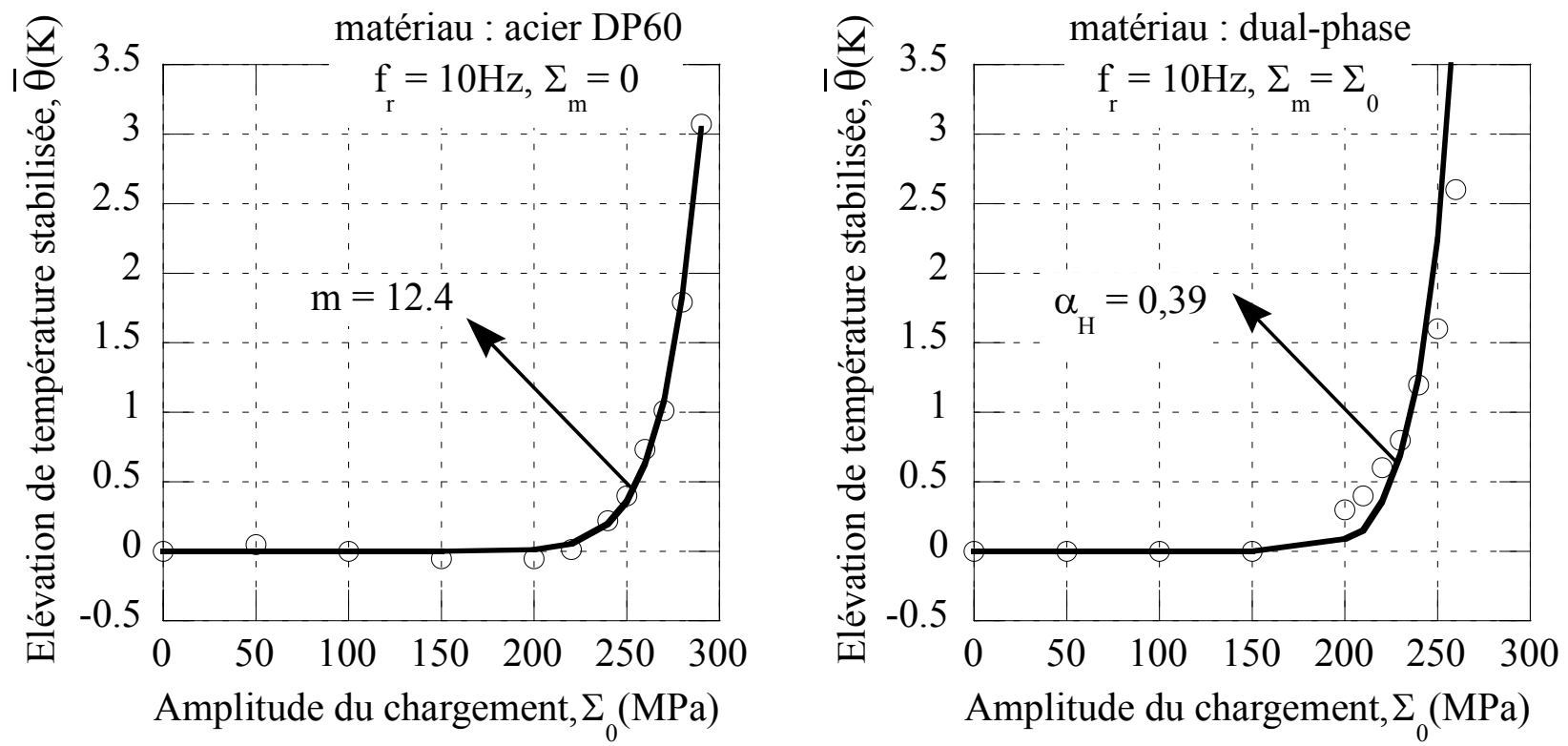

Fig. 7. Identification des paramètres à partir des courbes d'auto-échauffement pour : -a$\Sigma_{m}=0 M P a,-\mathrm{b}-\Sigma_{m}=\Sigma_{0}$.

Fig. 7. Identification of the parameters from self-heating tests for: $-a-\Sigma_{m}=0 M P a,-b-\Sigma_{m}=\Sigma_{0}$.

\begin{tabular}{cccc}
\hline$m$ & $\alpha_{H}$ & $V_{0}^{I / m} S_{0}$ & $A$ \\
\hline 12.4 & 0.39 & $438 \mathrm{MPa} . \mathrm{mm}^{3 / 12.4}$ & $1600 \mathrm{GPa}^{2}$ \\
\hline
\end{tabular}

Tableau 1. Paramètres pour l'acier dual-phase.

Table 1. Parameters for the dual-phase steel.

La validation du modèle est quant à elle effectuée, d'une part, sur la prévision de l'autoéchauffement, et d'autre part, sur la base de la prévision des résultats de campagnes d'essais de fatigue. Les figures $8 \mathrm{a}$ et $8 \mathrm{~b}$ montrent les comparaisons entre les résultats d'auto-échauffement et les prévisions du modèle, pour un autre rapport de charge et dans le cas de chargements multiaxiaux (i.e., surface d'iso-auto-échauffement). L'autoéchauffement est bien décrit par le modèle dans les deux cas. 

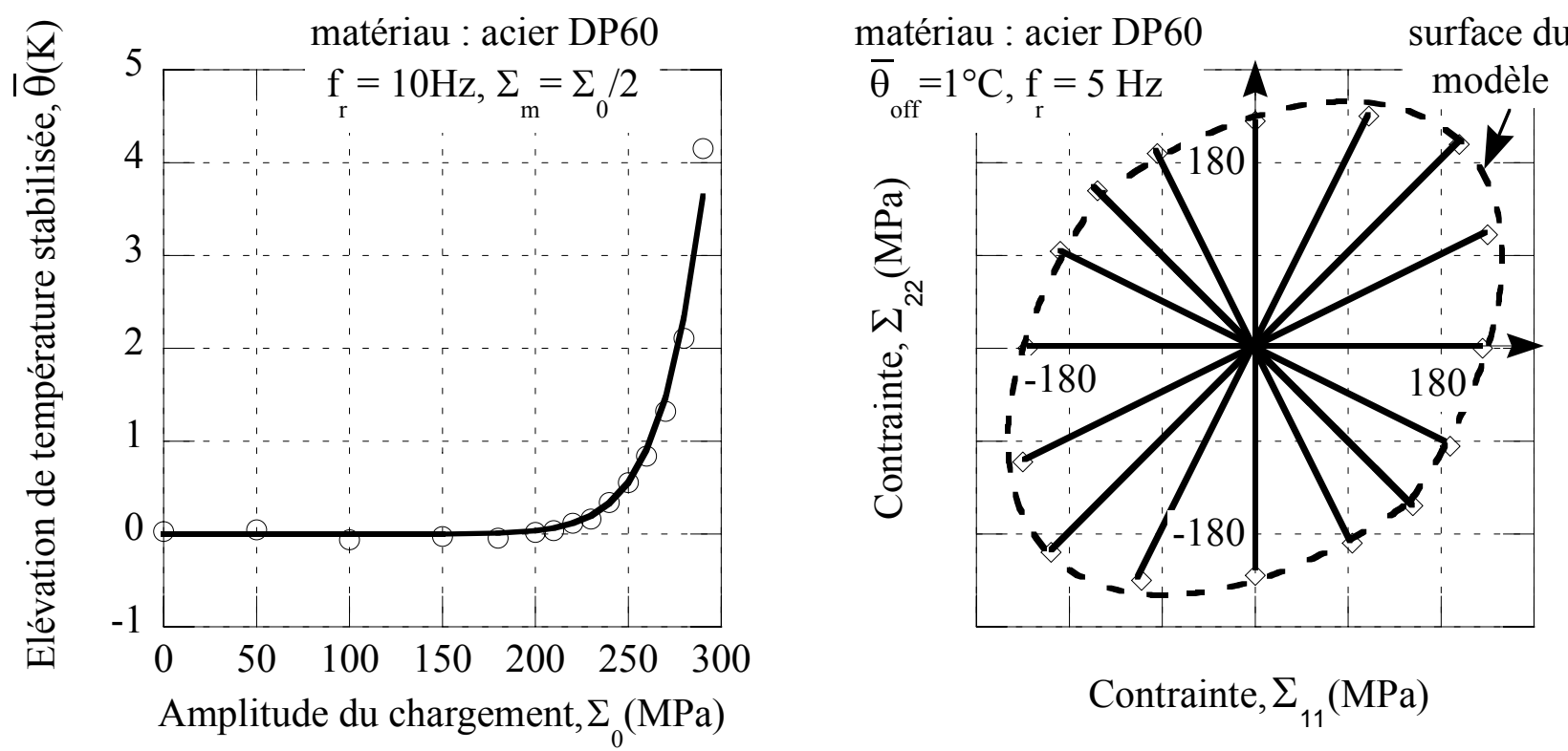

Contrainte, $\Sigma_{11}(\mathrm{MPa})$

Fig. 8. Prévision de l'auto-échauffement : -a- courbe d'auto-échauffement pour $\Sigma_{m}=0 M P a$, -bsurface d'iso-auto-échauffement.

Fig. 8. Prediction of the self heating: - a-steady state temperature for $\Sigma_{m}=\Sigma_{0} / 2,-b$ - isotemperature surface.

Les figures $9 \mathrm{a}$ et $9 \mathrm{~b}$ montrent les comparaisons entre les résultats d'essais de fatigue à différentes contraintes moyennes pour ce même acier et les prévisions du modèle. Un très bon accord est observé dans les deux cas.
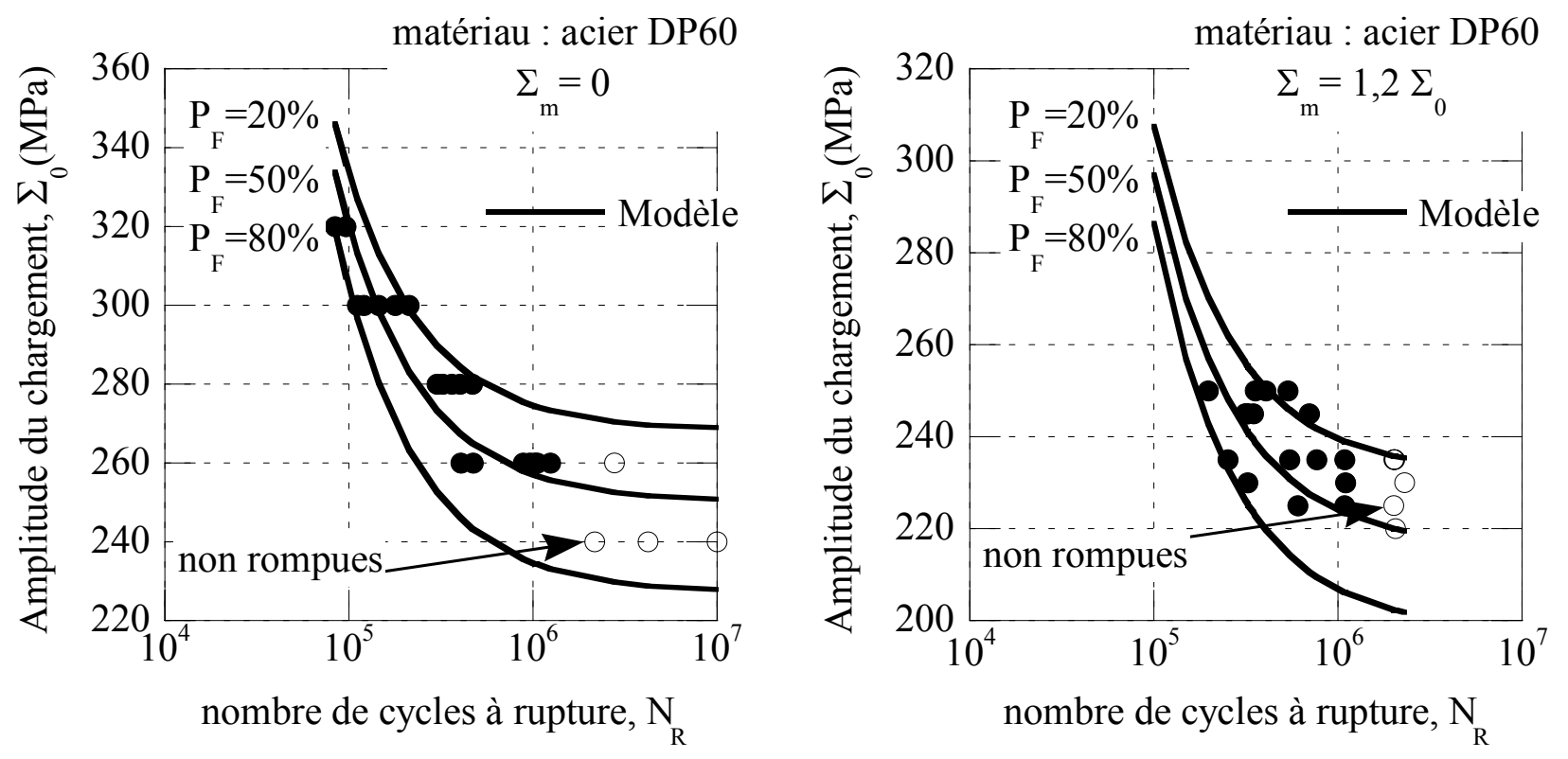

Fig. 9. Prévision des courbes de Wöhler pour différentes contraintes moyennes. -a- $\Sigma_{m}=0 M P a$, b- $\Sigma_{m}=1,2 \Sigma_{0}$.

Fig. 9. Prediction of the Wöhler curves for: $-a-\Sigma_{m}=0 M P a,-b-\Sigma_{m}=1,2 \Sigma_{0}$. 


\section{CONCLUSIONS}

Les essais d'auto-échauffement sous chargements cycliques apportent des informations précieuses pour le développement de modèles pour la fatigue à grand nombre de cycles des matériaux métalliques. Ils nous renseignent sur la nature des ingrédients à considérer dans la modélisation : scénario d'activation des sites dissipatifs, nature du comportement de ces sites, ... En ce qui concerne le cadre que nous proposons, une approche probabiliste à deux échelles basée sur un scénario d'apparition progressive de sites micro-plastiques a été retenue. Le modèle est identifié à partir d'essais rapides d'auto-échauffement (ne nécessitant que peu d'éprouvettes et peu de temps) à rapport de charge différent, mené jusqu'à rupture lors du dernier palier et permet de rendre compte des différentes observations expérimentales (effet de la fréquence, effet du rapport de charge, effet du caractère multiaxial, ...). Il est validé sur la base de la prévision des courbes de Wöhler du matériau et du comportement à l'auto-échauffement pour des chargements multiaxiaux.

\section{références}

[1] G. Welter (1937) "Essais d'endurance par traction et compression," Wlad. Inst. Met., 32 .

[2] M. P. Luong (1992) "Infrared Thermography of Fatigue in Metals," SPIE 1682, $222-233$.

[3] G. La Rosa and A. Risitano "Thermographic Methodology for Rapid Determination of the Fatigue Limit of Materials and Mechanical Components," (2000) Int. J. Fat. $22[1], 65-73$.

[4] P. K. Liaw, H. Wang, L. Jiang, B. Yang, J. Y. Huang, R. C. Kuo and J. C. Huang (2000) "Thermographic detection of fatigue damage of pressure vessel at $1 \mathrm{~Hz}$ and $20 \mathrm{~Hz}$," Scripta Materialia, 42 [4], 389-395. 
[5] A. Galtier, O. Bouaziz and A. Lambert (2002) "Influence de la Microstructure Des Aciers sur leurs Propriétés Mécaniques,” Méc. Ind. 3 [5], 457-462.

[6] C. Doudard, S. Calloch, F. Hild, P. Cugy and A. Galtier (2004) "Identification of the scatter in high cycle fatigue from temperature measurements," C.R. Mécanique 332 [10], 795-801.

[7] C. Doudard, S. Calloch (2009) "Influence of hardening type on self-heating of metallic materials under cyclic loadings at low amplitude," Eur. J. Mech. A/Solids 28, 233-240.

[8] C. Doudard, S. Calloch, P. Cugy, A. Galtier and F. Hild (2005) “A probabilistic two-scale model for high-cycle fatigue life predictions," Fat. Fract. Eng. Mat. Struct. 28, 279-288.

[9] C. Doudard, (2004) «Détermination rapide des propriétés en fatigue à grand nombre de cycles à partir d'essais d'échauffement, »Thèse de doctorat de l'ENS de Cachan.

[10] M. Poncelet (2007) « Multiaxialité, hétérogénéités intrinsèques et structurales des essais d'auto-échauffement et de fatigue à grand nombre de cycles, » Thèse de doctorat de l'ENS de Cachan.

[11] C. Doudard, M. Poncelet, S. Calloch, C. Boué, F. Hild, B. Weber, A. Galtier (2007) "Determination of an HCF criterion by thermal measurements under biaxial cyclic loading,” Int. J. Fat. 29, 748-757.

[12] M. Poncelet, C. Doudard, S. Calloch, F. Hild, B. Weber, A. Galtier (2007) "Prediction of self-heating measurements under proportional and non-proportional multiaxial cyclic loadings," C.R. Mécanique 335, 81-86. 
[13] R. Gulino and S. L. Phoenix (1991) "Weibull Strength Statistics for Graphite Fibres Measured from the Break Progression in a Model Graphite/Glass/Epoxy Microcomposite,” J. Mater. Sci. 26 [11], 3107-3118.

[14] M. Berveiller, A. Zaoui, An extension of the self-consistent scheme to plastically flowing polycrystals, J. Mech. Phys. Solids 26 (1979) 325-344.

[15] J. Lemaitre et J. Chaboche, Mécanique des matériaux solides, Dunod, 2004.

[16] A.M. Freudenthal, Statistical approach to brittle fracture, in: H. Liebowitz (Ed.), Fracture, vol. 2, Academic Press, New York, 1968, p. 591-619.

[17] W. Weibull, A statistical theory of the strength of materials, Roy. Swed. Inst. Eng. Res. 151 (1939). 